\title{
Optimized Task Scheduling Algorithms in Distributed Computing: A Review
}

\author{
Nidhi Tyagi \\ ABSS Engineering College, Meerut
}

\author{
Vinita Sharma \\ ABSS Engineering College, Meerut
}

\begin{abstract}
Cost is becoming the fruity factor to show the performance for any kind of experiment. Task scheduling algorithms are the valuable term in these days because of its performance in distributed computing environment. Market wants to pay minimum cost for every aspect. Task scheduling is performing the lead role in distributed computing with reducing cost. Various optimized task scheduling has been developed for execute the task efficiently. This paper proves that optimized task scheduling algorithms are better than traditional one's ever.
\end{abstract}

\section{Keywords}

Task scheduling, Cost, Distributed computing.

\section{INTRODUCTION}

A model, in which various elements of software are, passes to the various processors to perform better in parallel manner. Because of sharing, efficiency of the scheduling process is increases. Within the geographic area, elements are shared through distributed computing. Sharing can be done in between the elements and tasks as well. Parallel processing is improved by using the concept of distributed environment. Sharing can be performed anywhere through multiple systems. For business orientation process, distributed computing has outperformed in the technological world. The standard distribution system using 3-tier architecture, user interface dealing is execute in the processor at the specified region, marketing is works through a remote system, and database authority access and execution is designed in another system that arrange centralized approach for many big organization processing system. Distributed computing technique works on client/server architecture in standard way. Big industries are using distributed computing environment to make the availability of the services through this system for their client. Third party participates to do the communication to the server and client to provide the service through Internet. Third party works as a broker thought the whole communication. Broker will be responsible for any kind of inconvenience for any particular activity. Broker plays an important role for the whole communication for any activity. Broker stores all the per-requisites from the client and server as well, and then provide the required service with authentication process. None of the forgery will be entertained by client side that is take care by the broker side.

Many directions are working in a parallel manner in distributed environment. Scheduling is one of the directions in distributed real time environment. Scheduling maps the activity to the processor for required resources. Many optimized task scheduling is in working stage to improve the efficiency of the system. Optimization introduced with the help of the priority concept. Priorities has been set by improving the particular factor like cost, makespan, time complexity, load balancing, latency etc .

\section{TASK SCHEDULING ALGORITHMS}

Author improves the makespan by using probabilistic distribution approach. By calculating the threshold values, scheduling algorithm has been optimized.This optimization evade from the complex mathematics computation with random number of generated variables. [1]

Author presents an allocation model that share the valuable tasks among the entire processors in distributed computing environment by captivate the factors like: reducing the communication cost, load balancing and availability the required applications. [2]

Author introduces this algorithm by using two phases. First phase is set the priorities for the valuable tasks; second phase is the selection procedure for the particular task. For first phase, a priority queue is designed with introducing the key values with upper and lower rank. For queue implement, binary heap constructed. Initially each task is pointed as a critical task. Then selection phase is in working with critical path processor. On the other hand, assignment of the task enters in the processing, which reduces the earliest finish time for a particular task. [3]

Author designed task scheduling algorithm with implementing a genetic algorithm for allocation of task to search for an optimum solution to the specific problem. Cost is a very crucial factor in the field of technology. This paper reduces the execution cost for the entire process. Parallel processing has been performed for numerous tasks. Number of task must be greater than number of processors to perform better for real life application. [4]

Author elaborates the performance evaluation of various scheduling algorithms based on some specific parameters. It includes two types of load balancing approaches that are mathematical technique \& soft computing technique. Experimented analysis shows that algorithms have strength and weaknesses as well. More optimized scheduling can be developed in future to improve the technology. [5]

Author developed a new level that is used to recognize the priority for every activity in heterogeneous distributed computing. It works in three passes; first is to choose the activity, second choose the process and third updating state table. To accomplish the pass one, directed acyclic graph is designed for each processor. Task is allocated to the processor which helps to reduce its finish time execution in second pass. Status of the system is updated in third pass. [6]

Author explains the various optimized task scheduling algorithms in cloud computing environment and calculated the allocation cost factor for quality of service optimized task scheduling algorithm and shows the improved results with comparison of traditional task scheduling algorithms. [7]

Author proposed the probability measurement of the relationships between the complex measurement and various 
fields for performing a task execution in a distributed computing system. Trust factor has been introduced by using probability function. [8]

Author represents task scheduling in multiprocessor system. By creating a queue, a novel scheduling approach has been designed, which uses a searching algorithm that is breath first search. The proposed algorithm uses two queues. First is Ready Task Queue (RTQ) and second is Not Ready Task Queue (NRTQ). By applying precedence, these queues are computed. Priority has been designed by using RTQ. The proposed algorithm outperform with the experimented results in the technological world. [9]

Author developed a tabu search algorithm. It is a technique for searching best quality neighbor. It finds the global optimum solution. It keeps the record of already searched solution in order to avoid the repetition for searching another node. It checks for all the possible outcomes in all direction and then move towards the best direction by using tabu search optimization algorithm. [10]

Author approached a new algorithm that is static based approach. It performs well for both heterogeneous and homogeneous processor systems. Its objective is to obtain a good quality of scheduling. Proposed parallel bubble scheduling is a new approach, in which a task is divided into sub tasks and performs individually to get the solution. Proposed algorithm performs well with both periodic and nonperiodic graph structures with reduced cost factor. [11]

Author implemented an algorithm by using ant colony optimization. This proposed algorithm is capture by the nature of the ants. An ant moves in group always for searching food. This environment nature becomes an optimized task scheduling algorithm. A chemical released by the first ant and then group of ant follow that pheromone chemical to move ahead. Shortest path can be found with the help of this nature. [12]
Author designed a novel framework, and it broadcasts a real time dynamic trust. Third-party service level agreement keeps a dynamic real-time on-demand service establishment component. Service provider is in selection based on the calculated trust. A window-based monitoring is developed in this research; remove the conditions associated to the dynamic real world applications, by reducing the quantity of input set carried throughout the network. Evaluations have been done by fetching the feedback from the users. By using this monitoring model, exact feedbacks can be retrieved. [13]

Author proposed a honeybees task scheduling algorithm with reducing cost factor. Enhancement for the result author evaluated the performance with more number of tasks varying from $20-200$. In order to gain the reduced cost, performance metrics such as cost and time of basic honeybees and advance honeybees have been calculated as well as compared from traditional scheduling algorithm and found that the proposed algorithm performs better in terms of cost. For future more factors will be considered like trust, fault tolerance, minimum completion time for further enhancement. [14]

Author introduced a novel algorithm based on nature activity and compared to particle swam optimization. Cuckoo search perform very well and improve the system efficiency by using probability distribution functions (Cauchy, Gauss and Gamma) to measure the random walk step. This paper works in real time dynamic system. Three type of cuckoo search task scheduling implemented that are linear, exponential and power increasing switching parameters and tested against the constant. [15]

\section{PERFORMANCE ANALYSIS}

Though experimental evaluation, conclusion is that optimized task scheduling algorithm always perform better in technology. Technology is growing day by day with increasing the demand of the user daily. Users are becoming smart with the technology advancement. Table 1 shows the finding from existing task scheduling algorithm.

Table 1. Existing task scheduling algorithm

\begin{tabular}{|l|l|c|c|c|}
\hline S.N. & \multicolumn{1}{|c|}{ Algorithm } & Time & Cost & $\begin{array}{c}\text { Load } \\
\text { balancing }\end{array}$ \\
\hline 1 & Probability distribution approach & $\sqrt{ }$ & $\times$ & $\times$ \\
\hline 2 & Sharing approach & $\times$ & $\sqrt{ }$ & $\sqrt{ }$ \\
\hline 3 & Priority set for tasks & $\sqrt{ }$ & $\times$ & $\times$ \\
\hline 4 & Genetic algorithm & $\times$ & $\sqrt{ }$ & $\times$ \\
\hline 5 & Mathematical experiments & $\sqrt{ }$ & $\sqrt{ }$ & $\times$ \\
\hline 6 & Activity based priority & $\sqrt{ }$ & $\times$ & $\times$ \\
\hline 7 & Quality of service & $\times$ & $\sqrt{ }$ & $\times$ \\
\hline
\end{tabular}




\begin{tabular}{|c|c|c|c|c|}
\hline 8 & probability measurement & $\sqrt{ }$ & $\sqrt{ }$ & $x$ \\
\hline 9 & Queue based approach & $\sqrt{ }$ & $x$ & $x$ \\
\hline 10 & Tabu searching & $\sqrt{ }$ & $\sqrt{ }$ & $x$ \\
\hline 11 & $\begin{array}{l}\text { multiprocessor } \\
\text { problem }\end{array}$ & $x$ & $\sqrt{ }$ & $x$ \\
\hline 12 & $\begin{array}{lll}\text { Heuristic } & \text { Task } & \text { Scheduling } \\
\text { Algorithm } & & \end{array}$ & $\sqrt{ }$ & $\sqrt{ }$ & $x$ \\
\hline
\end{tabular}

\section{CONCLUSION}

In the world of technology, many scheduling algorithms are in existence. Researchers have done much research in the field of distributed computing with real time system. This paper discusses some of the tasks scheduling algorithms. Resultant table is stated that optimized techniques perform better in some specific condition with particular factor. All of them are compared with the traditional algorithms on the basis of introduced factors like execution time, cost and load balancing etc. Resultant table shows that the efficiency of every

optimization method is better than the traditional ones. For future work, reservation

and availability for resources can be done in order to make task scheduling algorithms more efficient.

\section{REFERENCES}

[1] Wei Z and Rizos S, "Stochastic DAG scheduling using a Monte Carlo approach", Journal of Parallel Distributed Computing, Vol.73 , pp. 1673-1689, 2013.

[2] Chu W.W., "Optimal File Allocation in a Multiple Computing System", IEEE Trans. on Computer, Vol.C18, pp.885-889, 1969.

[3] Yanyan D and Xiangli Z, "A Synthesized Heuristic Task Scheduling Algorithm", Hindawi Publishing Corporation The Scientific World Journal, 2014.

[4] Ahmed Younes. Hamed ,"Task Allocation for Minimizing Cost of Distributed Computing Systems Using Genetic Algorithms" International Journal of Advanced Research in Computer Science and Software Engineering, Vol.2, Issue 9, September 2012.

[5] Ruchi Gupta, P.K Yadav, "Mathematical modeling of load Distribution problem in distributed Computing Environment-A State of Art." International Journal of Advance Research in Computer Science Software Engineering, Vol.4 Issue 7, pp. 1106-1119, July 2014.

[6] Daoud MI, Nawwaf K, "A High Performance Algorithm For Static Task Scheduling In Heterogeneous Distributed Computing Systems", IEEE Transactions on Parallel Distributed Systems, Vol.28, 2007.
[7] Nidhi Bansal, Amitab Maurya, Tarun Kumar, Manzeet Singh and Shruti Bansal, Cost performance of Qos driven task scheduling in cloud computing, ELSEVIER, Procedia Computer Science, International Conference on Recent Trends in Computing, vol. 57, pp. 126-130, 2015.

[8] Jigyasu Dubey and Vrinda Tokekar, "Bayesian Network Based Trust Model with Time Window for Pure P2P Computing Systems", IEEE Global Conference on Wireless Computing and Networking, pp. 219-223, 2014.

[9] Ranjit R, “A Novel Approach for Task Scheduling in Multiprocessor System", International Journal of Computer Applications, Vol.44, 2012.

[10] Marcin Markowski, "Tabu-Search Algorithm for Optimization of Elastic Optical Network Based Distributed Computing Systems", Intelligent Data Engineering and Automated Learning, pp. 361-369, 2015.

[11] Ahmad, Ishfaq, and Yu-Kwong K, "On parallelizing the multiprocessor scheduling problem, "Parallel and Distributed Systems, IEEE Transactions, Vol.10, 1999.

[12] Yanyan D and Xiangli Z, "A Synthesized Heuristic Task Scheduling Algorithm", Hindawi Publishing Corporation The Scientific World Journal, 2014.

[13] Varalakshmi Perumal, Judgi Thangavel, Saranya Ramasamy and Swathy Harish, "Dynamic Trust Establishment and Amended Window based Monitoring in Cloud", IEEE International Symposium on Electronic System Design, pp. 162-166, 2013.

[14] Anjuli Garg and Dr. C. Rama Krishna, "An improved honey bees life scheduling algorithm for a public cloud", International Conference on Contemporary Computing and Informatics, pp. 1140-1147, 2015.

[15] Guosun Zeng, "Trusted Dynamic Scheduling for LargeScale Parallel Distributed System",IEEE International Conference on Parallel Processing Workshops, pp. 137144, 2011. 